\title{
Manifestações clínicas gastrointestinais em pacientes com COVID-19
}

Clinical gastrointestinal manifestations in patients with COVID-19

Manifestaciones clínicas gastrointestinales en pacientes con COVID-19

Larissa Ferreira Alves da Silva ORCID: https://orcid.org/0000-0001-5198-6752 Universidade Tiradentes, Brasil

E-mail: larissaferreirase@gmail.com

Danielle Simões Cardoso

ORCID: https://orcid.org/0000-0002-6103-3864 Universidade Tiradentes, Brasil

E-mail: daniellecardoso95.dsc@gmail.com

Laryssa Fialho de Oliveira Sousa ORCID: https://orcid.org/0000-0002-4710-4558 Universidade Tiradentes, Brasil

E-mail: laryssa.fialho@ souunit.com.br

Marianna Rodrigues Marques Dourado ORCID: https://orcid.org/0000-0002-7743-7396 Universidade Tiradentes, Brasil

E-mail: marianna.rodrigues@souunit.com.br

Pedro Henrique Santos de Jesus ORCID: https://orcid.org/0000-0002-3728-4567 Universidade Tiradentes, Brasil

E-mail: pedro.jesus00@ souunit.com.br Elaine Oliveira Lima

ORCID: https://orcid.org/0000-0002-2539-3782 Universidade Tiradentes, Brasil E-mail: eolima94@gmail.com

Horley Soares Britto Neto

ORCID: https://orcid.org/0000-0002-3141-7488 Universidade Tiradentes, Brasil E-mail: horleyneto2@gmail.com

Mariana Sattler Lima Medina ORCID: https://orcid.org/0000-0001-5615-4605 Universidade Tiradentes, Brasil

E-mail: mariana.medina24@gmail.com Luísa Teixeira Silveira ORCID: https://orcid.org/0000-0003-1325-3296 Universidade Tiradentes, Brasil

E-mail: luisa.tsilveiraa@gmail.com

Cynthia Gabrielle Sousa Rocha ORCID: https://orcid.org/0000-0002-6754-2758 Secretaria Municipal de Saúde de Aracaju, Brasil E-mail: cynthinhagabi@gmail.com

Ana Regia Oliveira de Andrade ORCID: https://orcid.org/0000-0002-6819-613X Secretaria Municipal de Saúde de Aracaju, Brasil E-mail: anaregiasoliveira@hotmail.com

Leda Maria Delmondes Freitas Trindade ORCID: https://orcid.org/0000-0003-4300-4274 Universidade Tiradentes, Brasil

\section{Resumo}

Objetivo: Identificar as manifestações clínicas do trato gastrointestinal em pacientes acometidos pela COVID-19 e monitorados pela Secretaria Municipal de Saúde de Aracaju. Metodologia: estudo retrospectivo, transversal, tipo survey, realizado no período de março a julho de 2020, a partir de informações contidas em banco de dados da Secretaria Municipal de Saúde de Aracaju. Amostra composta por 3.342 pacientes cadastrados no programa de telemonitoramento da Secretaria que foram testados e confirmados pelo RT-PCR para SARS-CoV-2. Dados analisados por meio de frequência percentual, testes de Pearson, Shapiro-Wilks e de Mann-Whitney. Nível de significância adotado de 5\%. Resultados e Discussão: foram registrados 938 (27,36\%) pacientes com manifestações gastrointestinais: diarreia 637 (18,58\%), dor abdominal 158 (4,61\%), náuseas 156 (4,55\%), vômitos 89 (2,6\%) e 
anorexia 119 (3,47\%). Sendo a idade média de 39,1 ( $\pm 14,8)$ anos, $563(60 \%)$ do sexo feminino e 930 (99,9\%) residentes na grande Aracaju. A idade média dos casos graves foi de 55,5 $( \pm 18,9)$ anos e 53,9\% pertenciam ao sexo masculino. A média de duração dos sintomas gastrointestinais foi de 6,5 dias. Todos os casos graves foram internados e, desses, $4(0,5 \%)$ tiveram óbito. Principais comorbidades associadas aos casos graves $(\mathrm{p}<0,05)$ : doenças cardiovasculares, diabetes mellitus, doença renal crônica, neoplasias e doenças neurológicas. Conclusão: percebeu-se associação importante entre sintomas gastrointestinais em pacientes com a COVID-19, especialmente em mulheres jovens. A presença de comorbidades prévias foi fator estatisticamente significativo para o aparecimento de manifestações do trato digestivo.

Palavras-chave: COVID-19; Sinais e sintomas digestórios; Trato gastrointestinal.

\begin{abstract}
Objective: To identify the clinical manifestations of the gastrointestinal tract in patients affected by COVID-19 and monitored by the Municipal Health Department of Aracaju. Methodology: retrospective, cross-sectional, survey-type study, carried out from March to July 2020, based on information contained in a database of the Health Department of Aracaju. Sample composed of 3,342 patients registered in the telemonitoring program of the Department, who were tested and confirmed by RT-PCR for SARS-CoV-2. Data analyzed using percentage frequency, Pearson, ShapiroWilks and Mann-Whitney tests. Adopted significance level of 5\%. Results and Discussion: 938 (27.36\%) patients with gastrointestinal manifestations were registered: diarrhea 637 (18.58\%), abdominal pain $158(4.61 \%)$, nausea 156 (4.55\%), vomiting $89(2.6 \%)$ and anorexia $119(3.47 \%)$. With a mean age of $39.1( \pm 14.8)$ years, $563(60 \%)$ were female and $930(99.9 \%)$ lived in Aracaju or nearby. The mean age of severe cases was $55.5( \pm 18.9)$ years and $53.9 \%$ were male. The average duration of gastrointestinal symptoms was 6.5 days. All severe cases were hospitalized and, of these, $4(0.5 \%)$ died. Main comorbidities associated with severe cases $(p<0.05)$ : cardiovascular diseases, diabetes mellitus, chronic kidney disease, cancer and neurological diseases. Conclusion: there was an important association between gastrointestinal symptoms in patients with COVID-19, especially in young and female patients. The presence of previous comorbidities was a statistically significant factor for the appearance of digestive tract manifestations.
\end{abstract}

Keywords: COVID-19; Signs and symptoms, Digestive; Gastrointestinal tract.

\begin{abstract}
Resumen
Objetivo: Identificar las manifestaciones clínicas del tracto gastrointestinal en pacientes afectados por COVID-19 y monitoreados por la Secretaría Municipal de Salud de Aracaju. Metodología: estudio retrospectivo, transversal, tipo encuesta, realizado de marzo a julio de 2020, con base en información contenida en una base de datos de la Secretaría Municipal de Salud de Aracaju. Muestra compuesta por 3.342 pacientes registrados en el programa de telemonitorización de la Secretaría que fueron evaluados y confirmados por RT-PCR para el SARS-CoV-2. Datos analizados mediante frecuencia porcentual, pruebas de Pearson, Shapiro-Wilks y Mann-Whitney. Nivel de significancia adoptado del 5\%. Resultados y Discusión: Se registraron $938(27,36 \%)$ pacientes con manifestaciones gastrointestinales: diarrea 637 (18,58\%), dolor abdominal 158 (4,61\%), náuseas $156(4,55 \%)$, vómitos 89 (2,6\%) y anorexia 119 (3,47\%). La edad media fue 39,1 ( \pm 14,8) años, $563(60 \%)$ eran mujeres y $930(99,9 \%)$ vivían en el Gran Aracaju. La edad media de los casos graves fue de 55,5 ( $\pm 18,9)$ años y el 53,9\% eran varones. La duración media de los síntomas gastrointestinales fue de 6,5 días. Todos los casos graves fueron hospitalizados y, de ellos, $4(0,5 \%)$ fallecieron. Principales comorbilidades asociadas a casos graves $(\mathrm{p}<0,05)$ : enfermedades cardiovasculares, diabetes mellitus, enfermedad renal crónica, neoplasias y enfermedades neurológicas. Conclusión: hubo una asociación importante entre los síntomas gastrointestinales en pacientes con COVID-19, especialmente en pacientes jóvenes y mujeres. La presencia de comorbilidades previas fue un factor estadísticamente significativo para la aparición de manifestaciones del tracto digestivo.
\end{abstract}

Palabras clave: COVID-19; Signos y síntomas digestivos; Tracto gastrointestinal.

\title{
1. Introdução
}

A nova doença pelo coronavírus (COVID-19) teve seu primeiro relato em dezembro de 2019 na província de Wuhan, China, e se disseminou rapidamente para outros países, acarretando sérias ameaças à saúde pública. Em razão da alta infectividade e da rápida disseminação do vírus por todo o mundo, a Organização Mundial da Saúde declarou uma pandemia de COVID-19 em março de 2020 (Grossberg, Pellish, Cheifetz, \& Feuerstein, 2020). Sabe-se que a COVID-19 é ocasionada pelo SARS-CoV-2, um vírus de RNA de fita simples do gênero de beta-coronavírus (Wong, Lui, \& Sung, 2020). Nesse contexto, semelhante ao que ocorre em outras infecções virais, o SARS-CoV-2 é predominantemente disseminado por meio de gotículas respiratórias ou aerossóis, embora relatos de transmissão da doença pelo contato com fômites contaminados também já foram descritos (Thevarajan, Buising, \& Cowie, 2020). 
Além disso, há indicativos de que o receptor da enzima conversora da angiotensina II (ECA II) desempenha um papel fundamental na entrada e na adesão viral às células hospedeiras do organismo humano (Schmulson, Dávalos, \& Berumen, 2020). Esses receptores são abundantes no trato gastrointestinal, o que sugere que o SARS-CoV-2 pode infectar e se replicar nos órgãos do sistema digestivo através de mecanismos que ainda vêm sendo elucidados (Mandal, Konala, Adapa, Naramala, \& Gayam, 2020). Evidências recentes indicam a possibilidade de transmissão oral-fecal do vírus, e esse dado suscita a necessidade de uma mudança rápida e eficaz nos mecanismos de triagem e diagnóstico (Brooks \& Bhatt, 2021).

O espectro clínico da COVID-19 é complexo e ainda não é totalmente conhecido. Desse modo, as manifestações clínicas típicas da doença são: febre, tosse seca, alterações do paladar ou do olfato, fadiga e dispneia (Iser et al., 2020). Embora as manifestações respiratórias agudas da doença sejam as mais comuns, os Centros de Controle e Prevenção de Doenças acreditam que os sintomas gastrointestinais também podem ser indicadores de infecção por COVID-19 (Elshazli et al., 2021). Sintomas do trato gastrointestinal são descritos em uma variedade de infecções respiratórias virais agudas e, durante a pandemia, devem ser intimamente associados à COVID-19. Sendo assim, qualquer indivíduo com uma sintomatologia associada ao trato digestivo sem explicação alternativa deve ser isolado e rastreado para a doença, visando diminuir, dessa forma, a sua disseminação (Ata, Almasri, Sajid, \&Yousaf, 2020).

A maioria das manifestações gastrointestinais associadas à COVID-19 são leves e autolimitadas e incluem anorexia, diarreia, náuseas, vômitos e dor ou desconforto abdominal. Uma minoria de pacientes apresenta abdome agudo com etiologias como pancreatite aguda, apendicite aguda, obstrução intestinal, isquemia intestinal, hemoperitônio ou síndrome do compartimento abdominal (Kariyawasam, Jayarajah, Riza, Abeysuriya, \& Seneviratne, 2021). Na prática clínica, já foram descritos casos com queixa de dor abdominal em região epigástrica de forte intensidade, acompanhada de vômitos e distensão abdominal, levando os pacientes a serviço de urgência (Ahmed et al., 2020; Valdes et al., 2020). Todavia, por serem menos frequentes, esses sintomas são difíceis de serem reconhecidos como manifestações da COVID-19. Sendo assim, as estratégias de prevenção e a descoberta precoce da doença são primordiais para cessar a transmissão do vírus (Poggiali, Ramos, Bastoni, Vercelli, \& Magnacavallo, 2020).

Anormalidades hepáticas também podem estar presentes em virtude da infecção viral nos hepatócitos, da toxicidade das drogas utilizadas durante o tratamento e do estado inflamatório sistêmico desencadeado pelo vírus (Mao et al., 2020). Desse modo, a maioria das lesões hepáticas são transitórias, podendo cursar com elevação discreta das enzimas hepáticas e da bilirrubina sérica; entretanto, danos graves ao fígado podem acontecer, principalmente em pacientes acometidos pela forma grave da COVID-19 (Wong et al., 2020). O papel significativo das manifestações clínicas gastrointestinais na evolução clínica da COVID-19 é preciso, mas persistem muitas lacunas de conhecimento sobre sua fisiopatologia, manejo e valor preditivo. As manifestações do TGI associadas à forma grave da doença têm implicações tanto para o atendimento ao paciente quanto para o controle de infecção (Menon et al., 2021).

Considerando a complexidade da infecção pelo coronavírus e, tendo como referência os dados catalogados pelo Programa "Monitoraju" da Secretaria Municipal de Saúde de Aracaju, no período de março a julho de 2020, este estudo teve como objetivo analisar o perfil epidemiológico, evolução e prognóstico de pacientes acometidos pela COVID-19, que apresentaram como manifestações clínicas sinais e sintomas relacionados ao trato gastrointestinal.

\section{Metodologia}

Trata-se de uma pesquisa quantitativa, uma vez que busca explicações objetivas para a associação da sintomatologia do aparelho digestório, em pacientes com COVID-19, com ênfase na análise de dados numéricos (Estrela, 2018). Trata-se, ainda, de um estudo retrospectivo, transversal, tipo survey, a partir de informações contidas em banco de dados do programa de monitoramento telefônico da Secretaria Municipal de Aracaju ("Monitoraju") durante o período de acompanhamento de 
pacientes diagnosticados com COVID-19, através do teste RT-PCR, em amostras de swab de nasofaringe. A amostra foi composta por 3.428 pacientes confirmados e cadastrados no telemonitoramento e acompanhados por contato telefônico diário, no período de março a julho de 2020, independentemente da idade e gênero, que apresentaram, como manifestação clínica da COVID-19, sinais e sintomas do trato gastrointestinal.

Foram considerados critérios de exclusão pacientes cadastrados que recusaram o monitoramento à distância e o atendimento por contato telefônico independente do motivo; além de pacientes monitorados, testados ou não para COVID-19, que apresentaram como manifestação clínica somente sinais e sintomas respiratórios, cardiovasculares, cutâneos, articulares, tromboembólicos, neurológicos e geniturinários. O instrumento de pesquisa foi composto pelas seguintes variáveis: dados pessoais (identificação, idade, profissão, procedência, endereço); dados clínicos (sinais e sintomas, tipo de exame realizado, resultado dos exames, comorbidades, período de monitoramento, tempo de duração dos sintomas e internação hospitalar).

As variáveis categóricas foram descritas por meio de frequência absoluta e relativa percentual. As variáveis contínuas foram descritas por meio de média, mediana, desvio padrão e intervalo interquartil. A hipótese de independência entre variáveis categóricas foi testada por meio do teste Qui-Quadrado de Pearson. A hipótese de aderência intra-classe das variáveis contínuas à distribuição normal foi testada por meio do teste de Shapiro-Wilks. Uma vez rejeitada, a hipótese de igualdade de medianas foi testada por meio do teste de Mann-Whitney. O nível de significância adotado foi de $5 \%$ e o software utilizado foi o R Core Team 2021 (Versão 4.1.0).

Este estudo foi submetido e aprovado pelo Comitê de Ética em Pesquisa da Universidade Tiradentes, sob o parecer $n^{\circ}$ 4.371.612.

\section{Resultados e Discussão}

Neste estudo, foi avaliada uma população formada por 3.428 pessoas com testagem positiva para COVID-19, através do RT-PCR, entre os meses de março a julho de 2020. Desse total, foram excluídos 86 pacientes que recusaram o monitoramento, resultando em $3.342(98,67 \%)$ pacientes efetivamente monitorados. A idade média foi de $39,9( \pm 15,2)$ anos e $1.932(56,46 \%)$ eram do sexo feminino. Residiam na grande Aracaju, que abrange os municípios de Aracaju, Barra dos Coqueiros, São Cristóvão e Nossa Senhora do Socorro, 3.370 (99,82\%) respondentes (Tabela 1).

Tabela 1: Perfil epidemiológico de pacientes infectados pelo SARS-CoV-2. Aracaju, 2020.

\begin{tabular}{lcccc}
\hline & N & \% & Média (DP) & Mediana (IIQ) \\
\hline Idade & 3389 & 98,9 & $39,9(15,2)$ & $38(30-49)$ \\
Sexo & & & & \\
Feminino & 1932 & 56,46 & \\
Masculino & 1490 & 43,54 & \\
Município de Residência & & & \\
Grande Aracaju & 3370 & 99,82 & \\
Demais interiores de Sergipe & 5 & 0,15 & \\
Outros estados & 1 & 0,03 & \\
Monitorado & 3342 & 98,67 & \\
\end{tabular}

Legenda: $\mathrm{n}$ - frequência absoluta. \% - frequência relativa percentual. DP - Desvio Padrão. IIQ - Intervalo Interquartil. Fonte: Dados da pesquisa (2020).

Do total da população estudada, $1.080(31,51 \%)$ pacientes apresentavam comorbidades prévias sendo mais prevalentes Hipertensão Arterial Sistêmica 584 (17,04\%), Diabetes Mellitus 225 (6,56\%) e Asma 112 (3,27\%). Quanto aos sinais e sintomas do TGI, $938(27,36 \%)$ pacientes apresentaram essas manifestações clínicas; sendo a diarreia, a dor 
abdominal, náuseas, vômitos e anorexia os principais sinais e sintomas encontrados. Esses pacientes foram monitorados pela equipe do programa "Monitoraju", durante um período variável de 14 a 21 dias, por meio de contato telefônico diário. O tempo de duração total dos sintomas gerais apresentados pelo paciente foi igual ao tempo de evolução da doença (do início até a resolução dos sintomas). $\mathrm{O}$ tempo de duração dos sintomas gastrointestinais foi identificado como aquele intervalo de evolução da doença em que o paciente apresentou sintomas do TGI associados ou não a outros sintomas gerais. Apenas 240 (7\%) pacientes informaram o tempo de duração dos sintomas gastrointestinais, que durou em média 6,5 $( \pm 5,5)$ dias (Tabela 2).

Dados demonstraram que portadores de COVID-19 podem referir desconforto abdominal na ausência de sintomas respiratórios. Acredita-se que a presença da enzima conversora de angiotensina II em diversos órgãos da cavidade abdominal tenha um papel fundamental na etiologia da dor abdominal relacionada à infecção pelo SARS-CoV-2, uma vez que o vírus se conecta aos receptores dessa enzima (Saeed et al., 2020). Hoje sabe-se que esses receptores são altamente expressos em células glandulares dos epitélios gástrico, duodenal e retal, o que suscita a hipótese de que a transmissão viral também pode ocorrer através da via fecal-oral (Fu, Qian, \& Fu, 2020).

Em pacientes infectados pelo SARS-CoV-2, a diarreia - ainda que considerada um sintoma atípico - também foi relativamente comum nesta análise, em concordância com dados encontrados na literatura. Apesar de ter sido inicialmente encontrada em uma pequena parcela de casos, é crescente o número de pacientes que referem essa queixa (D’amico, Baumgart, Danese, \& Peyrin-Biroulet, 2020).

Vários estudos retrospectivos, de relatos unicêntricos, revelaram que uma quantidade notável de pacientes com COVID-19 referia sintomas gastrointestinais - como náuseas, vômitos, dor abdominal e diarreia. Uma análise comparativa feita por Zhai et al. (2020) da literatura chinesa, constatou que, diante de estudos realizados por Wang e cols. (2020), entre 138 pacientes avaliados, 10,1\% referiram, como sintomas iniciais da doença, diarreia e náuseas, mesmo antes do aparecimento de febre e sintomas respiratórios. Estudo descritivo, transversal e multicêntrico realizado por Pan e cols. (2020), demonstrou que, de 204 pacientes infectados com COVID-19, 50,5\% foram admitidos no hospital com sintomas gastrointestinais, sendo os mais prevalentes: perda de apetite $(78,6 \%)$, diarreia $(34 \%)$, vômitos $(3,9 \%)$ e dor abdominal $(1,9 \%)$, semelhante aos resultados encontrados no presente artigo. 
Tabela 2: Características quanto à presença de comorbidades e manifestações clínicas (sinais e sintomas) em pacientes com COVID-19. Aracaju, 2020.

\begin{tabular}{|c|c|c|c|c|}
\hline & $\mathbf{N}$ & $\%$ & $\begin{array}{c}\text { Média } \\
\text { (DP) }\end{array}$ & $\begin{array}{c}\text { Mediana } \\
\text { (IIQ) }\end{array}$ \\
\hline Comorbidades & 1080 & 31,51 & & \\
\hline Cardiopatia & 70 & 2,04 & & \\
\hline HAS & 584 & 17,04 & & \\
\hline $\mathrm{DM}$ & 225 & 6,56 & & \\
\hline Obesidade & 25 & 0,73 & & \\
\hline Etilismo & 4 & 0,12 & & \\
\hline Tabagismo & 17 & 0,50 & & \\
\hline DPOC & 4 & 0,12 & & \\
\hline Asma & 112 & 3,27 & & \\
\hline DRC & 12 & 0,35 & & \\
\hline Neoplasia Ativa & 31 & 0,90 & & \\
\hline Doenças Neurológicas & 27 & 0,79 & & \\
\hline Doenças Psiquiátricas & 37 & 1,08 & & \\
\hline Outros & 288 & 8,40 & & \\
\hline Assintomático & 268 & 7,82 & & \\
\hline Tosse Seca & 1186 & 34,60 & & \\
\hline Tosse Produtiva & 115 & 3,35 & & \\
\hline Dispneia & 635 & 18,52 & & \\
\hline Febre & 1278 & 37,28 & & \\
\hline Cianose & 6 & 0,18 & & \\
\hline Odinofagia & 721 & 21,03 & & \\
\hline Mialgia & 963 & 28,09 & & \\
\hline Astenia & 842 & 24,56 & & \\
\hline Artralgia & 103 & 3,00 & & \\
\hline Cefaleia & 1692 & 49,36 & & \\
\hline Congestão nasal/Coriza & 763 & 22,26 & & \\
\hline Tontura & 145 & 4,23 & & \\
\hline Confusão Mental & 4 & 0,12 & & \\
\hline Alterações cutâneas & 56 & 1,63 & & \\
\hline Disgeusia & 646 & 18,84 & & \\
\hline Anosmia & 695 & 20,27 & & \\
\hline Diarreia & 637 & 18,58 & & \\
\hline Dor Abdominal & 158 & 4,61 & & \\
\hline Náuseas & 156 & 4,55 & & \\
\hline Vômitos & 89 & 2,60 & & \\
\hline Anorexia & 119 & 3,47 & & \\
\hline Outros & 943 & 27,51 & & \\
\hline Manifestações do TGI & 938 & 27,36 & & \\
\hline Tempo de duração dos sintomas gastrointestinais & 240 & 7,0 & $6,5(5,5)$ & $5(2-11)$ \\
\hline Tempo de duração total dos sintomas apresentados & 1372 & 40,0 & $13(4,6)$ & $13(10-16)$ \\
\hline
\end{tabular}

Fonte: Dados da pesquisa (2020).

Dentre os pacientes que apresentaram sintomatologia relacionada ao TGI, a idade média encontrada foi de 39,1 $( \pm 14,8)$ anos, $563(60 \%)$ eram mulheres e 930 (99,9\%) residiam na Grande Aracaju. Além disso, ao comparar os pacientes com COVID-19 que apresentaram sintomatologia clínica relacionada ao trato digestivo e os que não apresentaram esses sintomas durante a evolução da doença, a maioria dos que tiveram acometimento do TGI apresentava comorbidades prévias, totalizando 341 pacientes $(\mathrm{p}<0,001)$. Dentre as comorbidades identificadas, observou-se que, percentualmente, $24(2,6 \%)$ eram 
cardiopatas, $41(4,4 \%)$ tinham diagnóstico de asma (p=0,026), $181(19,3 \%)$ de hipertensão arterial (p=0,031), 41 (4,4\%) eram diabéticos, $13(1,4 \%)$ tinham alguma doença psiquiátrica e $12(1,3 \%)$ doenças neurológicas $(\mathrm{p}=0,046)($ Tabela 3$)$.

Tabela 3: Comparativo entre os pacientes infectados pelo SARS-CoV-2 que apresentaram manifestações do TGI e os que não apresentaram essas manifestações clínicas. Aracaju, 2020.

\begin{tabular}{|c|c|c|c|}
\hline & \multicolumn{2}{|c|}{ Manifestações do TGI } & \multirow[b]{2}{*}{ p-valor } \\
\hline & Sim & Não & \\
\hline Idade, Média $(D P)$ & $39,1(14,8)$ & $40,3(15,3)$ & $0,049^{\mathrm{M}}$ \\
\hline Mediana $(I I Q)$ & $38(29-48)$ & $39(30-50)$ & \\
\hline \multicolumn{4}{|l|}{ Sexo } \\
\hline Feminino & $563(60)$ & $1369(55,1)$ & $0,010^{\mathrm{Q}}$ \\
\hline Masculino & $375(40)$ & $1115(44,9)$ & \\
\hline \multicolumn{4}{|l|}{ Município de Residência } \\
\hline Grande Aracaju & $930(99,9)$ & $2440(99,8)$ & $0,105^{\mathrm{Q}}$ \\
\hline Demais interiores de Sergipe & $0(0)$ & $5(0,2)$ & \\
\hline Outros estados & $1(0,1)$ & $0(0)$ & \\
\hline Monitorado & $931(100)$ & $2411(98,2)$ & $<0,001^{Q}$ \\
\hline Comorbidades & $341(36,4)$ & $739(29,7)$ & $<0,001$ Q \\
\hline Cardiopatia & $24(2,6)$ & $46(1,8)$ & 0,189 Q \\
\hline HAS & $181(19,3)$ & $403(16,2)$ & $0,031 \mathrm{Q}$ \\
\hline DM & $41(4,4)$ & $158(6,3)$ & $0,401 \mathrm{Q}$ \\
\hline Obesidade & $8(0,9)$ & $17(0,7)$ & $0,602 \mathrm{Q}$ \\
\hline Etilismo & $1(0,1)$ & $3(0,1)$ & $0,916^{\mathrm{Q}}$ \\
\hline Tabagismo & $4(0,4)$ & $13(0,5)$ & $0,722^{Q}$ \\
\hline DPOC & $1(0,1)$ & $3(0,1)$ & $0,916^{\mathrm{Q}}$ \\
\hline Asma & $41(4,4)$ & $71(2,9)$ & $0,026^{\mathrm{Q}}$ \\
\hline DRC & $4(0,4)$ & $8(0,3)$ & $0,642^{Q}$ \\
\hline Neoplasia Ativa & $7(0,7)$ & $24(1)$ & $0,549^{Q}$ \\
\hline Doenças Neurológicas & $12(1,3)$ & $15(0,6)$ & $0,046^{Q}$ \\
\hline Doenças Psiquiátricas & $13(1,4)$ & $24(1)$ & $0,286^{Q}$ \\
\hline Outros & $95(10,1)$ & $193(7,8)$ & $0,025^{\mathrm{Q}}$ \\
\hline
\end{tabular}

Legenda: $\mathrm{n}$ - frequência absoluta. \% - frequência relativa percentual. DP - Desvio Padrão. IIQ - Intervalo Interquartil. M - Teste de MannWhitney. Q - Teste Qui-quadrado de Pearson. HAS - Hipertensão Arterial Sistêmica. DM - Diabetes Mellitus. DPOC - Doença Pulmonar Obstrutiva Crônica. DRC - Doença Renal Crônica.

Fonte: Dados da pesquisa (2020).

Casos graves foram definidos como aqueles que necessitaram de internação hospitalar (4,4\%) e/ou resultaram em óbito $(0,5 \%)$. Somado a isso, $142(15,1 \%)$ pacientes relataram que fizeram uso de medicação (p<0,001) (Tabela 4). O número de pessoas em uso de medicação, entretanto, pode ser subestimado, uma vez que não foi um questionamento feito ativamente pelos profissionais responsáveis pelo monitoramento. Além disso, a internação hospitalar foi um fator que dificultou a manutenção do contato telefônico diário.

Uma meta-análise envolvendo nove estudos, provenientes de bancos de dados chineses e ingleses, avaliou a correlação entre a gravidade da COVID-19 e a presença de sintomas gastrointestinais durante a evolução clínica da doença. Ao comparar a gravidade da infecção pelo coronavírus entre pacientes com e sem sintomas do TGI, a análise não demonstrou diferença estatisticamente significativa entre os dois grupos (Liu, Cui, Yang, \& Yao, 2020). 
Tabela 4: Características quanto à evolução clínica da amostra avaliada. Aracaju, 2020.

\begin{tabular}{|c|c|c|c|}
\hline & \multicolumn{2}{|c|}{ Manifestações do TGI } & \multirow[b]{2}{*}{ p-valor } \\
\hline & Sim & Não & \\
\hline Internação Hospitalar & $41(4,4)$ & $121(4,9)$ & $0,548^{\mathrm{Q}}$ \\
\hline Uso de Medicação & $142(15,1)$ & $225(9)$ & $<0,001{ }^{Q}$ \\
\hline \multicolumn{4}{|l|}{ Desfecho secundário } \\
\hline Vida & $798(99,5)$ & $2169(99,2)$ & $0,358^{Q}$ \\
\hline Óbito & $4(0,5)$ & $18(0,8)$ & \\
\hline Grave & $41(4,4)$ & $126(5,1)$ & $0,403^{\mathrm{Q}}$ \\
\hline
\end{tabular}

Legenda: Q - Teste Qui-quadrado de Pearson.

Fonte: Dados da pesquisa (2020).

A Tabela 5 apresenta as características gerais dos casos graves da COVID-19 em pacientes que tiveram manifestações gastrointestinais durante a evolução da infecção. Dentre os pacientes que apresentaram a forma grave da doença, a idade média foi de 55,5 $( \pm 18,9)$ anos, com ligeiro predomínio no sexo masculino $(53,9 \%)$ em comparação com o sexo feminino $(46,1 \%)$.

A maior parte $(61,1 \%)$ desses pacientes apresentava comorbidades prévias $(\mathrm{p}<0,001)$ e a presença de cardiopatias, Hipertensão Arterial Sistêmica, Diabetes Mellitus, Doença Renal Crônica, neoplasias em atividade ou doenças neurológicas foi um fator estatisticamente importante na análise $(\mathrm{p}<0,05)$. Ademais, nas formas graves, o tempo de duração dos sintomas do TGI foi menor em comparação aos casos leves, sendo, em média, igual a 2,3 $( \pm 1,5)$ dias (p=0,046) enquanto que os casos leves tiveram em média 6,6 $( \pm 5,5)$ dias (Tabela 5). 
Tabela 5: Características gerais dos casos graves da COVID-19 com manifestações do TGI. Aracaju, 2020.

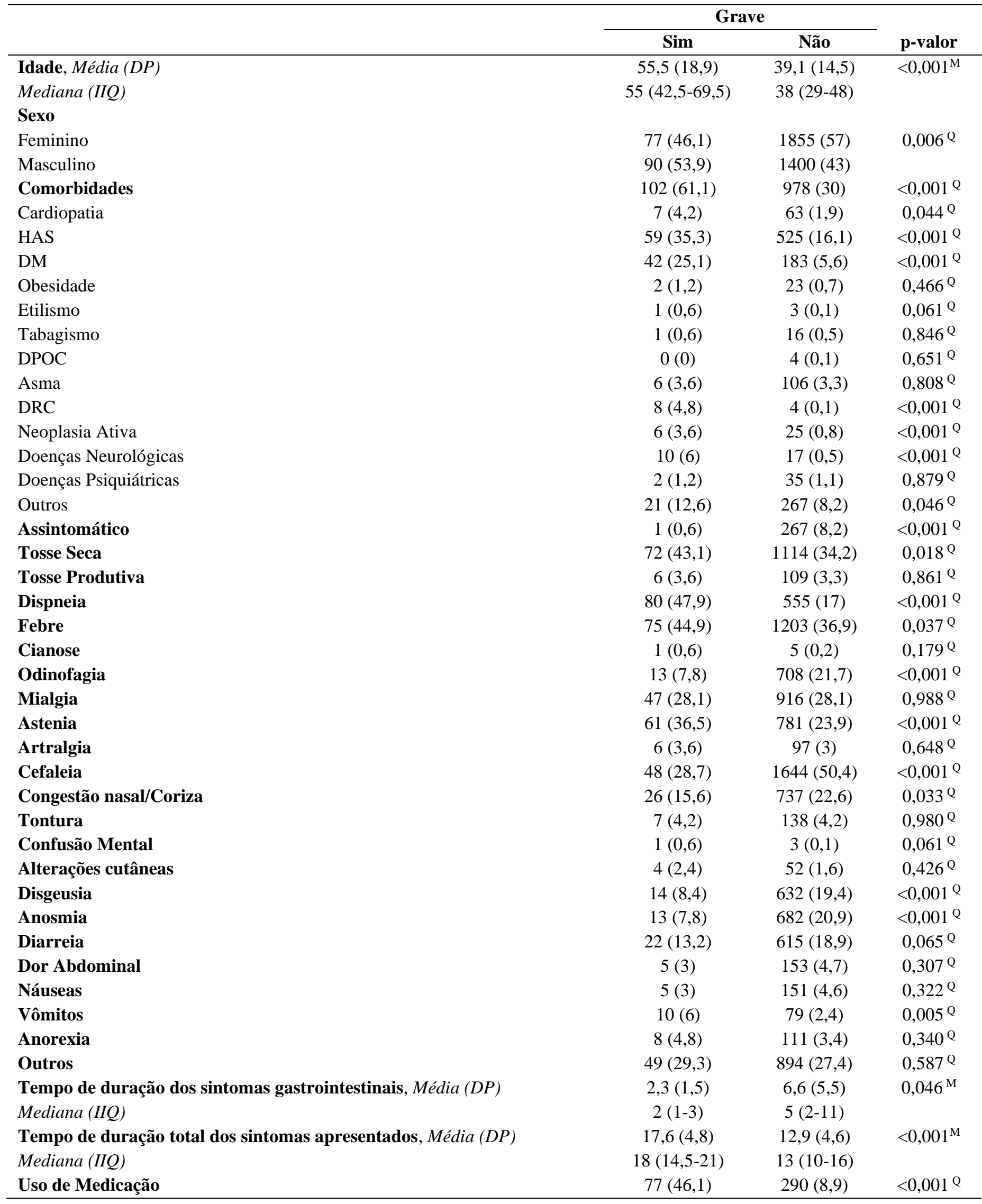

Legenda: $\mathrm{n}$ - frequência absoluta. \% - frequência relativa percentual. DP - Desvio Padrão. IIQ - Intervalo Interquartil. M - Teste de MannWhitney. Q - Teste Qui-quadrado de Pearson.

Fonte: Dados da pesquisa (2020). 


\section{Conclusão}

Os sintomas do aparelho digestivo podem passar despercebidos como manifestações iniciais na clínica da infecção pela COVID-19. Apesar de a apresentação clínica da doença constituir-se tipicamente de manifestações respiratórias tal qual ocorre em síndromes gripais, na população estudada, constatou-se uma associação estatisticamente relevante entre os sinais e sintomas do aparelho digestivo em pacientes com a COVID-19, com ligeira predominância em pacientes mais jovens e do sexo feminino.

A presença de comorbidades prévias se mostrou um fator estatisticamente significativo para o aparecimento de manifestações do trato gastrointestinal; apesar disso, essa sintomatologia não teve relação importante com os casos graves da doença nesta análise. Todavia, por se tratar de uma base de dados provenientes de monitoramento telefônico, a necessidade de internação hospitalar prejudicou o estabelecimento do contato diário e, assim, a caracterização precisa da sintomatologia apresentada nesses pacientes, o que se tornou um fator a ser considerado como limitação deste estudo.

Esse fato corrobora com a urgente necessidade de estabelecimento de ensaios clínicos bem desenhados para melhor entendimento e detecção das manifestações gastrointestinais na evolução clínica da COVID-19, assim como do desenvolvimento de estrutura e incentivos que permitam o monitoramento de casos confirmados para, assim, possibilitar a criação de protocolos eficazes no controle e manejo da doença.

\section{Referências}

Ahmed, A. O. E., Mohamed, S. F., Saleh, A. O., Al-Shokri, S. D., Ahmed, K., \& Mohamed, M. F. H. (2020). Acute abdomen-like-presentation associated with SARS-CoV-2 infection. IDCases, 21, e00895. 10.1016/j.idcr.2020.e00895

Ata, F., Almasri, H., Sajid, J., \& Yousaf, Z. (2020). COVID-19 presenting with diarrhoea and hyponatraemia. BMJ Case Reports, 13(6), e235456. $10.1136 /$ bcr-2020-235456

Brooks, E. F., \& Bhatt, A. S. (2021). The gut microbiome: a missing link in understanding the gastrointestinal manifestations of COVID-19? Molecular Case Studies, 7(2), a006031. 10.1101/mcs.a006031

D’Amico, F., Baumgart, D. C., Danese, S., \& Peyrin-Biroulet, L. (2020). Diarrhea During COVID-19 Infection: Pathogenesis, Epidemiology, Prevention, and Management. Clinical Gastroenterology and Hepatology, 18(8), 1663-1672. 10.1016/j.cgh.2020.04.001

Elshazli, R. M., Kline, A., Elgaml, A., Aboutaleb, M. H., Salim, M. M., Omar, M., Munshi, R., Mankowski, N., Hussein, M. H., Attia, A. S., Toraih, E. A., Settin, A., Killackey, M., Fawzy, M. S., \& Kandil, E. (2021). Gastroenterology manifestations and COVID-19 outcomes: A meta-analysis of 25,252 cohorts among the first and second waves. Journal of Medical Virology, 93(5), 2740-2768. 10.1002/jmv.26836

Freire, M; \& Pattussi, M. (2018). Tipos de estudo In Estrela, C. (Org.), Metodologia Científica: Ciência, Ensino, Pesquisa (pp. 187-188). São Paulo: Editora Artes Médicas.

Fu, B., Qian, K., \& Fu, X. (2020). SARS-CoV-2-Induced Vomiting as Onset Symptom in a Patient with COVID-19. Digestive Diseases and Sciences, 65(6), 1568-1570. 10.1007/s10620-020-06285-4

Grossberg, L. B., Pellish, R. S., Cheifetz, A. S., \& Feuerstein, J. D. (2020). Review of Societal Recommendations Regarding Management of Patients With Inflammatory Bowel Disease During the SARS-CoV-2 Pandemic. Inflammatory Bowel Diseases. 10.1093/ibd/izaa174

Iser, B. P. M., Sliva, I., Raymundo, V. T., Poleto, M. B., Schuelter-Trevisol, F., \& Bobinski, F. (2020). Definição de caso suspeito da COVID-19: uma revisão narrativa dos sinais e sintomas mais frequentes entre os casos confirmados. Epidemiologia e Serviços de Saúde, 29(3). 10.5123/s1679-49742020000300018

Kariyawasam, J. C., Jayarajah, U., Riza, R., Abeysuriya, V., \& Seneviratne, S. L. (2021). Gastrointestinal manifestations in COVID-19. Transactions of The Royal Society of Tropical Medicine and Hygiene. 10.1093/trstmh/trab042

Liu, J., Cui, M., Yang, T., \& Yao, P. (2020). Correlation between gastrointestinal symptoms and disease severity in patients with COVID-19: a systematic review and meta-analysis. BMJ Open Gastroenterology, 7(1), e000437. 10.1136/bmjgast-2020-000437

Mandal, A., Konala, V. M., Adapa, S., Naramala, S., \& Gayam, V. (2020). Gastrointestinal Manifestations in COVID-19 Infection and Its Practical Applications. Cureus. 10.7759/cureus. 8750

Mao, R., Liang, J., Shen, J., Ghosh, S., Zhu, L.-R., Yang, H., Wu, K.-C., \& Chen, M.-H. (2020). Implications of COVID-19 for patients with pre-existing digestive diseases. The Lancet Gastroenterology \& Hepatology, 5(5), 425-427. 10.1016/s2468-1253(20)30076-5

Menon, T., Sharma, R., Earthineni, G., Iftikhar, H., Sondhi, M., Shams, S., Khurshid Ahmed, N., Khan, H., Rathore, S. S., \& Singh, R. (2021). Association of Gastrointestinal System With Severity and Mortality of COVID-19: A Systematic Review and Meta-Analysis. Cureus. 10.7759/cureus.13317 
Research, Society and Development, v. 10, n. 13, e423101321542, 2021

(CC BY 4.0) | ISSN 2525-3409 | DOI: http://dx.doi.org/10.33448/rsd-v10i13.21542

Poggiali, E., Ramos, P. M., Bastoni, D., Vercelli, A., \& Magnacavallo, A. (2020). Abdominal Pain: A Real Challenge in Novel COVID-19 Infection. European Journal of Case Reports in Internal Medicine, 7(4). 10.12890/2020_001632

Saeed, U., Sellevoll, H. B., Young, V. S., Sandbaek, G., Glomsaker, T., \& Mala, T. (2020). Covid-19 may present with acute abdominal pain. British Journal of Surgery, 107(7), e186-e187. 10.1002/bjs.11674

Schmulson, M., Dávalos, M. F., \& Berumen, J. (2020). Alerta: los síntomas gastrointestinales podrían ser una manifestación de la COVID-19. Revista de Gastroenterología de México, 85(3), 282-287. 10.1016/j.rgmx.2020.04.001

Thevarajan, I., Buising, K. L., \& Cowie, B. C. (2020). Clinical presentation and management of COVID -19. Medical Journal of Australia, 213(3), 134-139. $10.5694 / \mathrm{mja} 2.50698$

Valdes, J. A. M. et al. Perforated Gastric Ulcer Related to SARS Cov-2 (COVID-19). EC Gastroenterology and Digestive System, 7(8), 144-152.

Wong, S. H., Lui, R. N., \& Sung, J. J. (2020). Covid-19 and the digestive system. Journal of Gastroenterology and Hepatology, 35(5), 744-748. $10.1111 /$ jgh. 15047

Zhai, L.-L., Wang, W., Wu, L., \& Tang, Z.-G. (2020). COVID-19 with abdominal symptoms and acute abdominal pain: a guide to identification for general practice. British Journal of General Practice, 70(696), 358-359. 10.3399/bjgp20x710693 